\title{
Design of intelligent tracking robot
}

\author{
Yibo Liu ${ }^{1 *}$, Ying Sun ${ }^{1}$, Du Jiang ${ }^{1}$ Juntong Yun${ }^{1}$, Ying Liu ${ }^{1}$, Dongxu Bai ${ }^{1}$, \\ Gongfa Li ${ }^{1}$, Dalin Zhou ${ }^{2}$
}

1.Key Laboratory of Metallurgical Equipment and control Technology of ministry of Education, Wuhan Un iversity of Science and Technology, Wuhan, Hubei,China

Wuhan University of Science and Technology, Wuhan, Hubei,China

Eamil:840877291@qq.com

2.School of Computing, University of Portsmouth, Portsmouth PO1 3HE, UK

\begin{abstract}
With the trend of world trade entering a period of rapid growth, the logistics industry is developing very rapidly. At the same time, it also has higher requirements for logistics handling and work efficiency. Therefore, this article designs a robot with mechanical arm and claw crawler tracking for unmanned logistics warehouse and production workshop. The robot has the functions of detecting signals, identifying signals, autonomously grasping objects, and transporting objects. Intelligent tracking robot is an industrial robot can be automated handling operations can be flexible grasping and handling of materials. The robot can automatically tracking, self-grasping objects, independent transport objects. Compared with the traditional crawler transport robot, the robot has the characteristics of less sensors, high accuracy, sensitive response and good adaptability. This article details the robot design process and functional module analysis.
\end{abstract}

Keywords:Crawler robot; handling robot; mechanical arm; autonomous tracking

\section{Introduction}

With the gradual implementation of our country's "manufacturing power" strategy and the vigorous development of Internet technology and artificial intelligence, all walks of life are developing in the direction of automation and intelligence. Although the logistics industry has maintained rapid growth in recent years, the overall operating efficiency is still low. Our country's logistics expenditure dropped from $23.79 \%$ in 1991 to $16.9 \%$ in 2019. The proportion of logistics costs in the United States and Japan in GDP is stable at about $8 \%$, the difference between my country and the 
United States and Japan is over $8 \%$ in this respect. This shows that the proportion of logistics costs in GDP has gradually declined, and it is very important to reduce costs. At the same time, according to the statistics of the China Federation of Logistics and Purchasing, in 2018, the total national social logistics was 283.1 trillion yuan, an increase of $6.4 \%$ year-on-year.Sub-quarter view, a quarter of 62.4 trillion yuan, an increase of $7.2 \%$; first half of the 131.1 trillion yuan, an increase of $6.9 \%$; the first three quarters of 204.1 trillion yuan, an increase of 6.7 percent. [1]. This shows that my country's total logistics volume has grown steadily, demand has jumped, and improving efficiency has become a problem we must solve. In traditional logistics operations, human handling takes a long time, and the work intensity of personnel is relatively high. At the same time, the efficiency of personnel sorting is low, and the relatively high error rate cannot effectively meet the characteristics of a large number of modern logistics handling categories [2]. On the other hand, as China gradually enters an aging society, the working-age population is getting less and less, and the newly-increased working population is worried about this laborious and boring porter work, which causes the labor cost of porters to increase. The higher. Therefore, the current logistics industry urgently needs intelligent logistics equipment, using automation technology and artificial intelligence technology to improve handling efficiency.

Intelligent handling robot designed in this paper can realize the functions of automatically grabbing the goods, following the track and automatically unloading the goods. It involves computer, sensing, information, communication and automatic control technologies, and is a typical high-tech complex. Intelligent handling robot uses the combination of Brasa main control board and Bigfish expansion board as a controller, uses four gray sensors for detection and identification, four DC motors drive tracking, and the motor and steering gear are directly powered by the main control board. The mechanical arm claw is driven by a servo motor. Wherein the processor core is a control board Brasa ATmega328, it has 14 digital input / output port; Bigfish LED dot matrix expansion board expansion, the interface servo motor, DC motor and the universal expansion interface. The intelligent handling robot in this article is designed to combine the two important requirements of handling goods and autonomously planning routes. The robotic arm can carry goods within a certain quality. Different ends can be installed to meet different requirements of handling to complete various shapes and workpiece transfer operating state, can be widely used loading and unloading machine tools, stamping machines automated production lines, automatic assembly lines, stacking handling, automatic handling containers and the like, greatly reduces human heavy physical labor, has broad market prospect.

In the subsequent chapters of this article, we will introduce the mechanical structure of this design, rationalize the design of the robot's kinematic structure, car body, mechanical arm, and mechanical claw; analyze and design each module of the robot when it working. 


\section{Related work}

Handling robots are industrial robots that can carry out automated transfer operations. They are also called unmanned transfer vehicles or AGVs. The earliest handling robot appeared in the United States in 1960, and Versatran and Unimate robots were used for handling operations for the first time [3]. The handling operation refers to holding the workpiece with one kind of equipment, which means moving from one processing position to another processing position. The handling robot can be installed with different end effectors to complete the work of handling workpieces of various shapes and conditions, which greatly reduces the heavy manual labor of human beings. At present, there are more than 100,000 handling robots used in the world, which are widely used in automatic loading and unloading of machine tools, automatic production lines of stamping machines, automatic assembly lines, palletizing, and containers [4]. Some developed countries have developed and implemented policies to limit the maximum amount of manual operation, beyond the limits of the operation must be done by a robot [5].

Many countries in the world are actively conducting research and development design of intelligent mobile robots. Mobile robots are an important branch of robotics that appeared in the 1960s. At that time, Nils Nilssen and Charles Rosen of the Stanford Research Institute developed the autonomous mobile robot named shakey from 1966 to 1972 . The purpose was to apply artificial intelligence technology in complex environments to complete autonomous reasoning of robot systems., planning and control [6]. Since then, mobile robots have grown from scratch, and the number has been increasing. As an important branch of mobile robots, intelligent mobile robots have also received more and more attention.

\section{3 integrated system design}

The intelligent handling robot is required to have an automatic tracking function, and black lines are laid on the floor workshop. The feedback of the detection signals of the black lines by the gray sensor controls the speed and direction of the body movement. In addition to the straight line tracking, there are also typical cross-point routes and The T-shaped intersection route to ensure the complete structure of the tracking route needs to consider and process the signal recognition. In addition to tracking requirements, the mechanical arm claws must be able to accurately grasp objects and transport objects, and different grasping mechanical grips can be replaced according to different object shapes and sizes.

1) Tracking requirements

The tracking function mainly includes straight-line driving, turning, T-shaped intersection turning, and cross intersection turning. Wherein the straight running claim robot can quickly and accurately identify the signal and can quickly be adjusted, but considering the actual situation, the motor cannot be too fast when the robot is driving in a straight line, otherwise it will cause the body to swing too much and will affect 
the preparation for the obstacle. Turning, T-junction turning, and cross-turn turning have strict requirements on the accuracy and response speed of the robot's identification system, and considering the placement of obstacles on the site, the robot body should not be too long or too fast, otherwise it will turn. The radius is too large, and some turns will be blocked by obstacles, which will cause the phenomenon that the body of the turn cannot be locked.

2) Crawl requirements

The manipulator claws can be required to have greater degrees of freedom and can be rotated in 360 degrees. You can put the objects into the cargo box at the rear of the body smoothly after grabbing, and you can take the objects out of the cargo box and put them in the designated position.

\section{$4 \quad$ Mechanical structure design}

\subsection{Selection of motion mechanism of intelligent robot}

In the structural design of intelligent robots, the kinematic mechanism is the first step in the journey from a thousand miles. If an intelligent robot does not have a suitable kinematic mechanism, then it cannot move unconstrained in its application environment. The kinematic mechanism is very large The degree determines the motion performance of the intelligent robot. The motion structure of the intelligent robot can be roughly divided into wheeled walking mechanism, crawler walking mechanism, leg walking mechanism, compound walking mechanism, etc. These types of mechanisms have different advantages and disadvantages, we need to consider the working environment of the intelligent robot to choose the most suitable movement mechanism.

(1) Wheel sports mechanism 


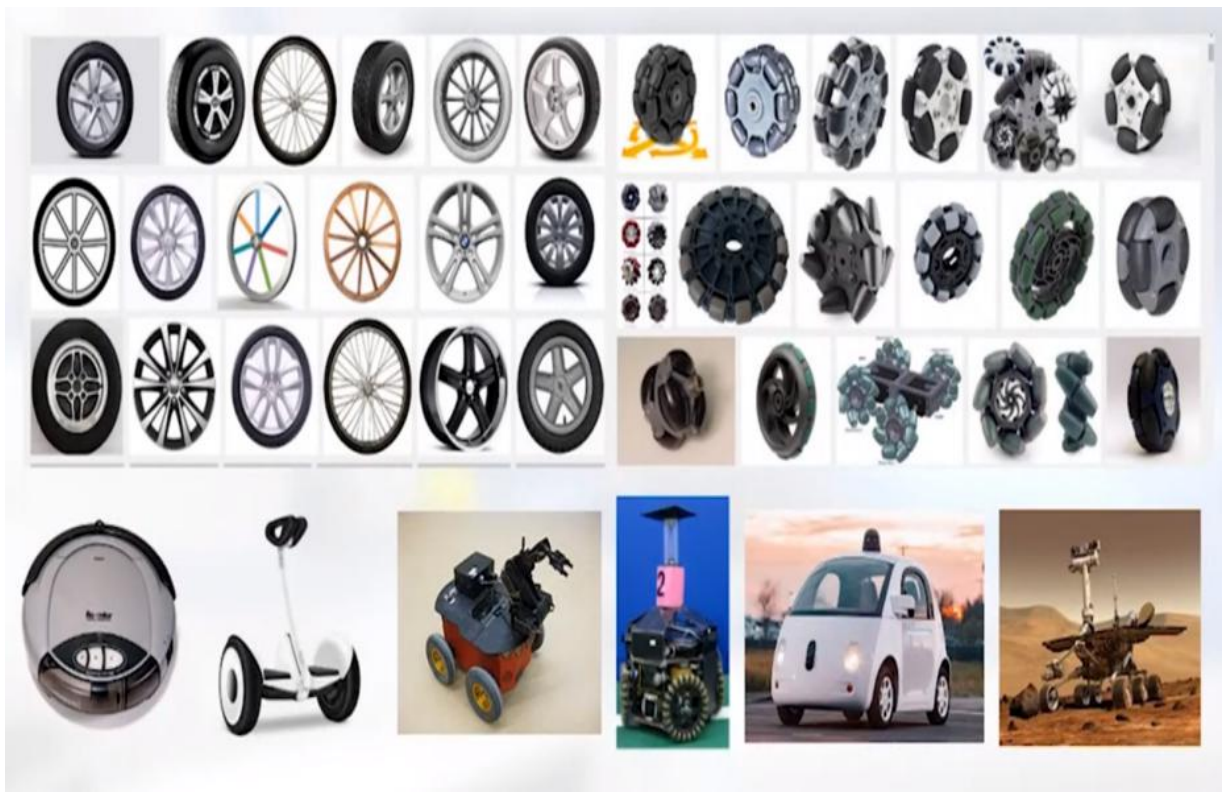

Fig.1 Wheel sports mechanism diagram

The active power wheel is one of the greatest inventions in human history. His appearance has brought huge changes to people's travel methods and field work, and also improved efficiency. He has extremely high sports efficiency on flat ground [7].

Compared with leg-type and crawler-type motion mechanisms, wheel-driven robots move faster and consume less energy; from a control point of view, wheel-type robots are relatively easy to control because of their simple mechanical structure and good stability. However, the requirements for the sports ground are high, and it is difficult to exercise in uneven and rough environments.

(2) Leg mobile mechanism

Leg-driven robots mostly adopt human-like or animal-like structures, and nature prefers leg-type movements. Like us, humans can move freely on various complex terrains such as grasslands and mountain streams with two legs. Insects are also because of their unique multi-leg structure Adhere to complex objects. However, the power selection and mechanical characteristics of the leg drive structure are very complicated, and the number of legs will also increase the difficulty of the mechanical design and the complexity of control [8].

(3) Crawler sports structure 


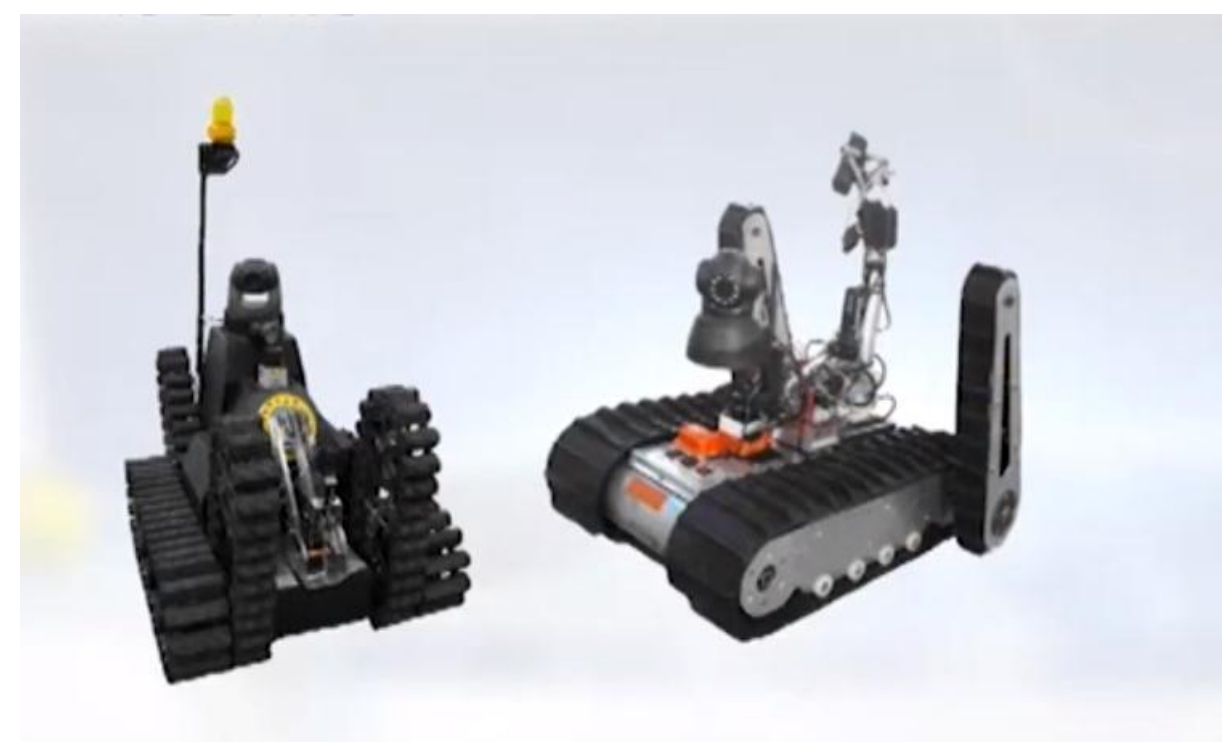

Fig.2 Crawler drive robot

The tracked sports structure is also a driving method that we can often see. Like tanks, tracked vehicles, etc., such sports structures are used. Its essence is a set of connecting rods connected to driving gears, wheels or sprockets. Allow them to run in a similar manner to the conveyor belt along the robot's chassis [9].

Crawler-type sports structure can provide greater traction, greater acceleration, better balance force than wheeled and leg type, and better obstacle-crossing ability; but the crawler may fail, and the drive mechanism may also occur The possibility of stuttering is relatively more difficult to repair.

After comparing the advantages and disadvantages of several walking mechanisms, combined with the requirements of the handling robot for traction, balance performance, and obstacle-crossing capabilities, this article selects the crawler walking mechanism as the movement mechanism of the handling robot.

\subsection{Main body and drive structure}

Main body using four drive DC motor drive with a direct current motor fixed to the bracket feet struts, the struts and feet fixed to the vehicle body, shown in Figure 3. Designed in a symmetrical way can effectively ensure the stability of the body. And because of the simplification of the design, the main body has a strong adaptability and excellent retrofitting performance. It can avoid making relatively large changes to the body structure later. 


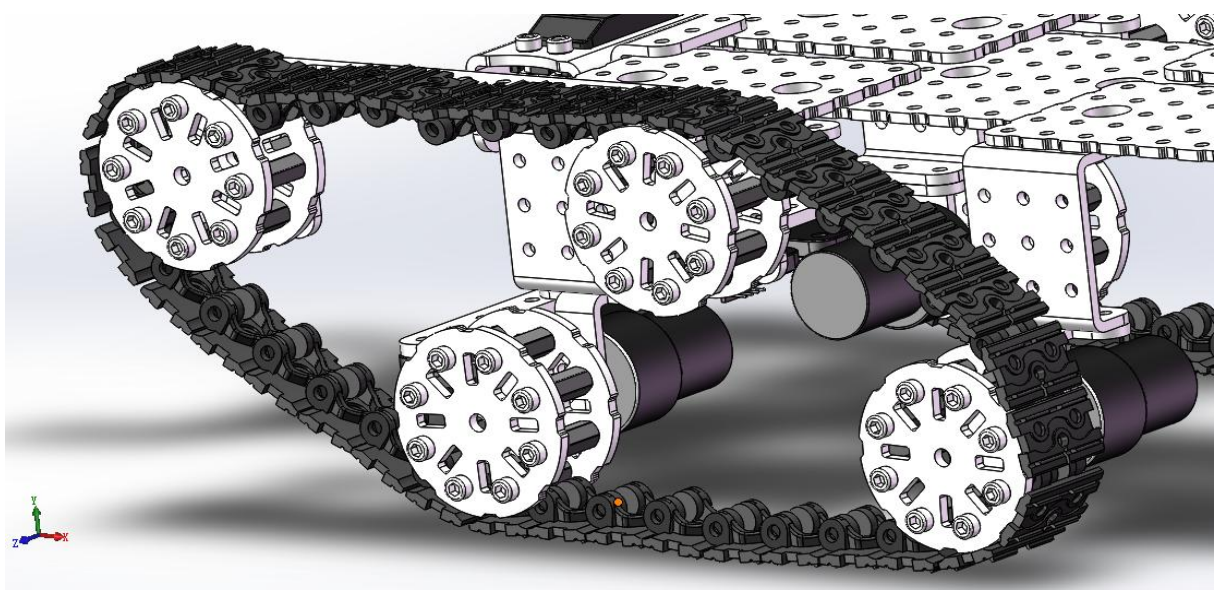

Fig3.Drive motor layout structure diagram

The whole adopts the crawler structure, and the overall body structure is shown in Figure 4.

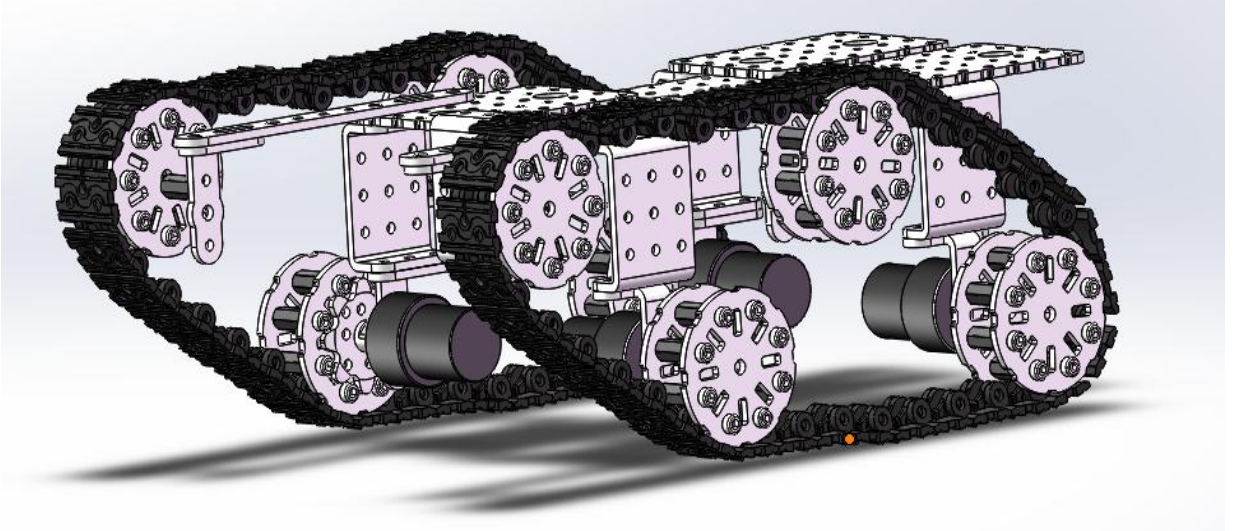

\subsection{Main tracking function structure}

Fig4. Schematic diagram of the overall body structure

Considering the realization of the tracking program, the robot use four gray sensors to be installed on the front rod of the vehicle body in a straight line, and it is connected to the bracket through screws, studs and connecting rods. Because the recognition distance of the sensor is short, the installation location is low. If you need to increase the distance, you can replace other sensors, such as white label sensors. Figure 5 shows the installation structure of the grayscale sensor. 


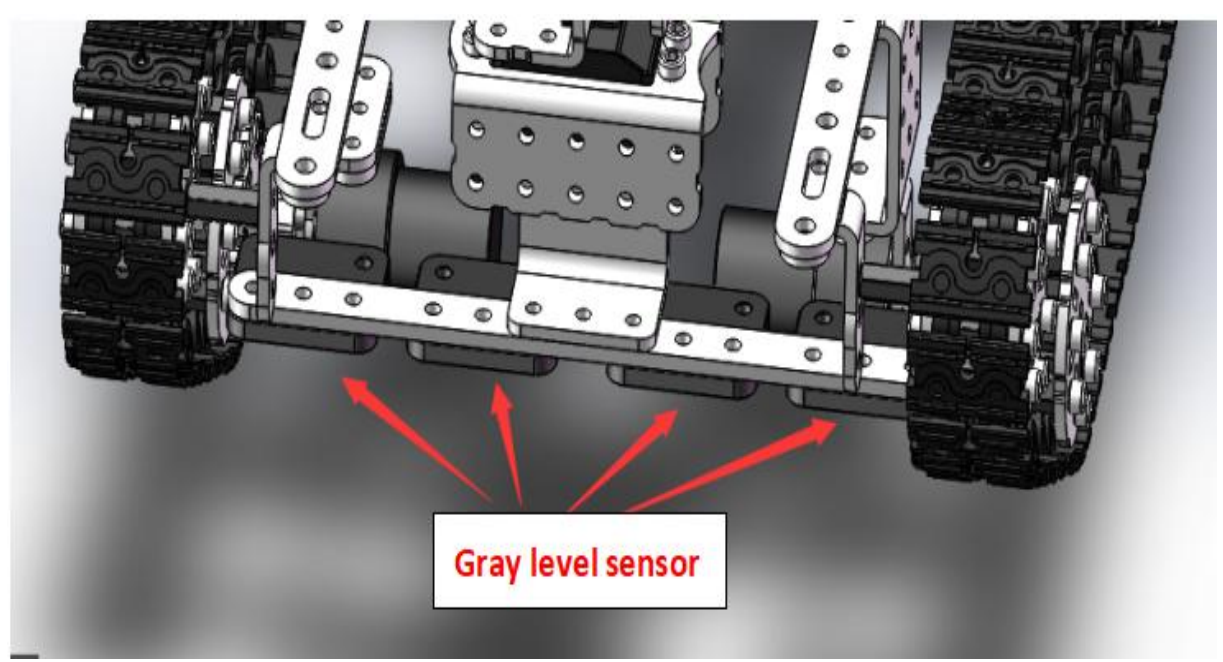

Fig 5.Gray level sensor structure

As the sensor detection system of the tracking module, the grayscale sensor is numbered 1234 sequentially from left to right, and the purpose is to detect. The level data is collected, and the intermediate value 2.5 is subtracted to determine whether the robot deviates from the established trajectory to the detection signal and control it.

\subsection{Manipulator claw structure}

Mechanical arm structure. The design requires a high degree of freedom for the robotic arm. A 3-degree-of-freedom mechanical arm structure is designed. The power is driven by a servo motor, which can accurately control the rotation angle, as shown in Figure 6.

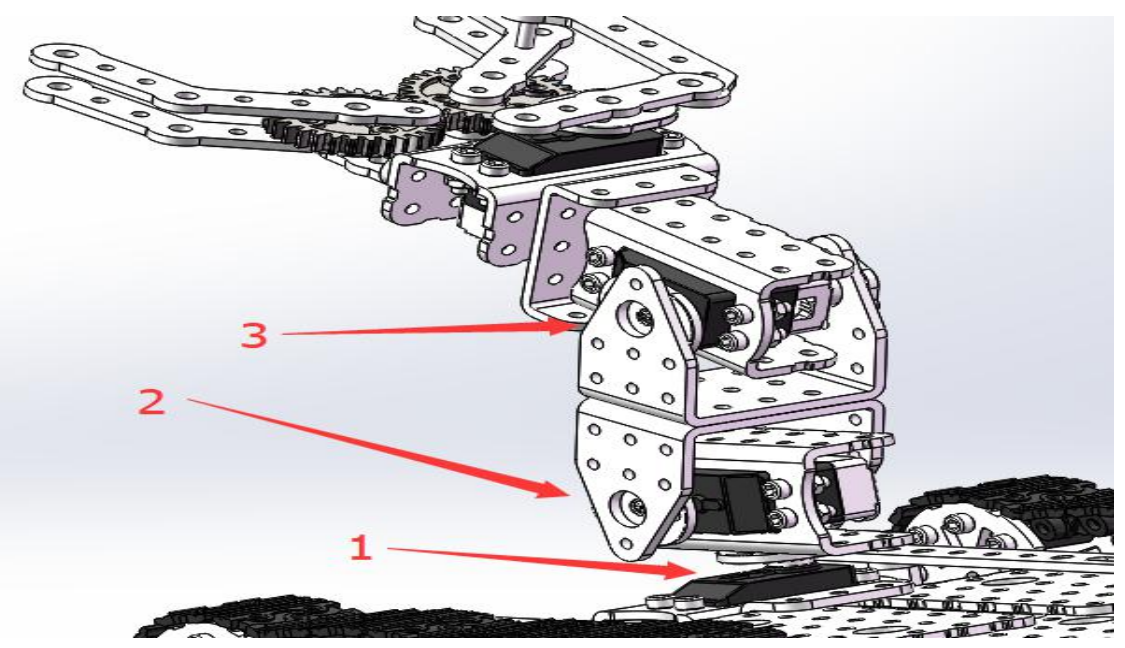

Fig6. Mechanical arm structure 
In Figure 6, the serial numbers of 1, 2, and 3 represent the movable parts of the robot arm respectively. Among them, the freedom of rotation around the $\mathrm{Z}$ axis of No. 1 can make the robot arm rotate $360^{\circ}$ around the vehicle body, and the number of 2 and 3 indicate that it can rotate around X. Degree of freedom of the axis or Y axis swing.

Mechanical claw structure.The function of the mechanical claw is to hold objects, which requires stable holding performance and high efficiency. The mechanical structure of the mechanical claw is shown in Figure 7.

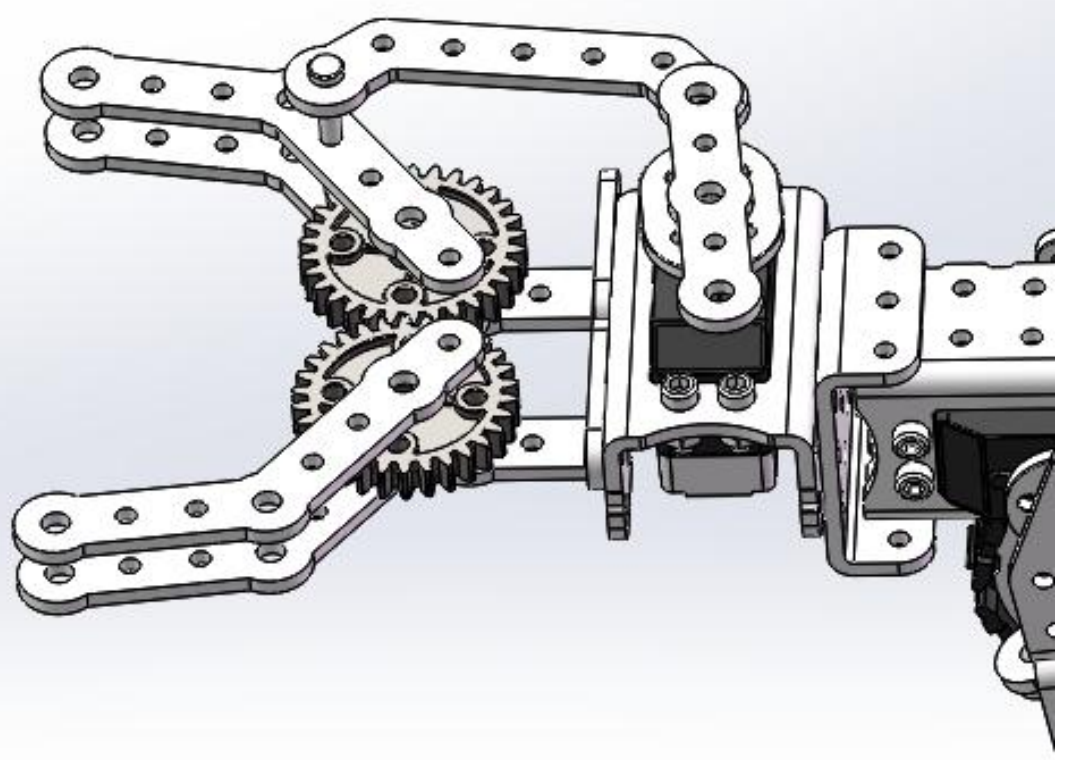

Fig7.Mechanical claw structure

Gripper driving force is provided by a servo motor, two fingers are connected by an intermediate gear transmitting the clamping force, of opening and closing can be done, hold the article stably.

\section{$5 \quad$ Intelligent handling robot module design}

According to the design idea and the consideration of the robot function, the robot includes five modules: gray sensor module, near infrared sensor module, speed module, tracking module, grab module, and power module. The following article will introduce the basic design of the five modules. 


\subsection{Gray sensor module:}

The gray level sensor is an analog sensor, with a light-emitting diode and a photoresistor, installed on the same surface. The gray level sensor uses different detection colors to reflect different levels of light, and the photoresistor uses different principles to detect the color depth of light returned by different detection surfaces. Within the effective detection distance, the light-emitting diode emits white light, illuminating the detection surface, the detection surface reflects part of the light, and the photoresistor detects the intensity of this light and converts it into a signal that can be recognized by the robot [10]. In this module, the collected information is processed to determine the current trend of the road where the intelligent handling robot is located and the information about the tilt of the robot body on the track. Pass the processed information to the control board to take corresponding control decisions for tracking requirements.

The article chooses the SEN1665 gray scale sensor as the tracking sensor to guide the robot. The sensor outputs a high-level signal with an amplitude of $5 \mathrm{~V}$, and the chip allows an input level of 3.3V. Similarly, the chip SN74LCX245 performs level conversion, and its circuit design is shown in the figure [11].

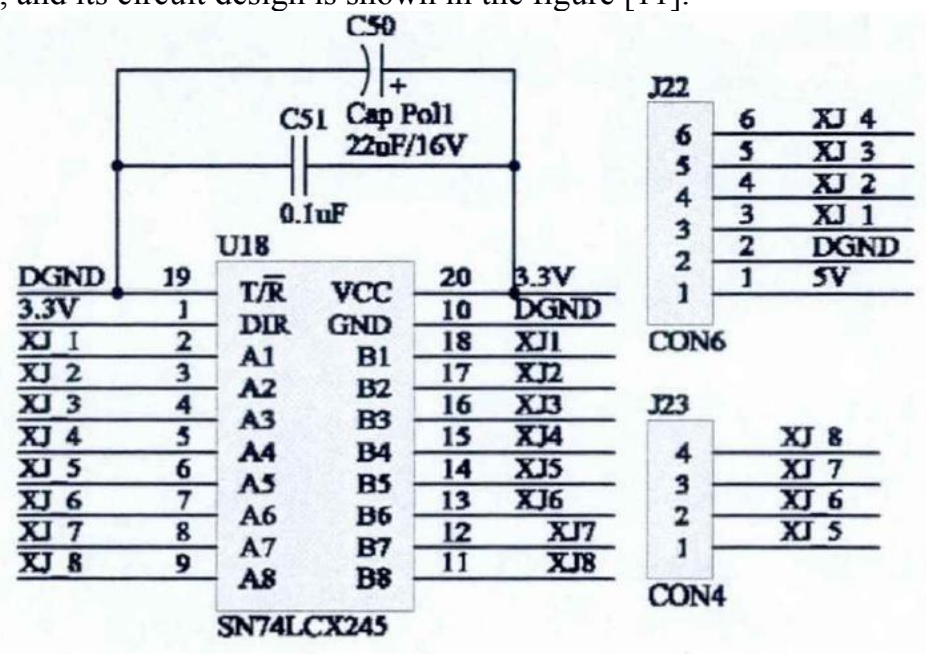

\subsection{Speed module}

Fig8. Gray level sensor interface diagram

Due to the instability of the main control board voltage supply and the error of the DC motor itself, it is difficult for the robot to maintain the two wheel speeds equal in actual conditions. Therefore, a PID algorithm is required to control the robot's movement direction and speed. But the effect of PID control mainly depends on three fixed parameters. When the parameters are fixed, they cannot be changed during the entire control process. The PID control technology is mature and the structure is simple, but the handling robot often has to work on goods of different weights, which 
cannot meet the actual control needs of the robot. Therefore, a fuzzy PID control algorithm is used to perform closed-loop control on the motor speed, thereby controlling the robot speed [12]. The following figure is the block diagram of fuzzy PID control system.

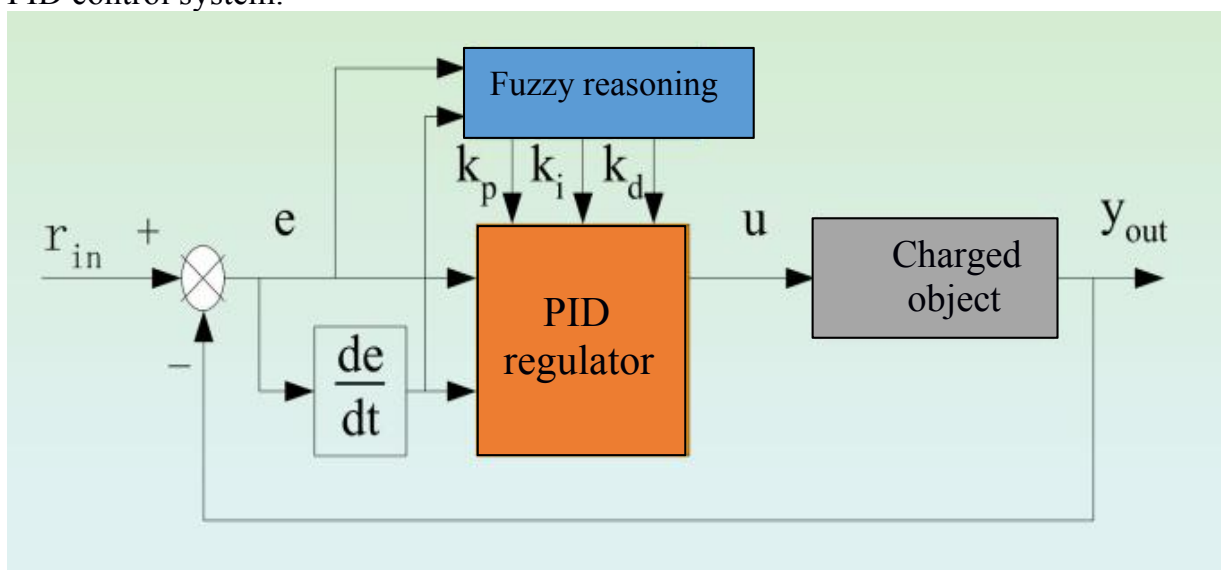

Fig9.Control block diagram of fuzzy adaptive PID system

Use infrared encoder to collect the current motor speed as the feedback of the system speed control, and at the same time compare with the set target speed, select the appropriate algorithm to make it change to the target speed, so as to achieve the acceleration and deceleration of the motor. Has good site adaptability and stability.

\subsection{Tracking module}

Tracking roughly divided into three steps First, the self-position information collection, and then calculated according to the signal obtained by the sensor output digital gray level signal, it is determined whether the shift position of the robot, if the offset would be corrected. Guide lines are set on the path where the robot moves. The guide lines are divided into black guide lines and white guide lines. If the gray sensor detects a black guide line, it outputs a high level, otherwise it outputs a low level. Finally, the tracking signal is processed. According to the value returned by the gray sensor, the offset of the robot relative to the wire position is obtained after statistical processing. First read the gray value high level, count the number of high levels, and take the average to make a difference with 2.5. If the deviation is 0 , it means that the robot center is on the black tracking line. If the deviation is less than 0 , the robot moves to the right, If the deviation $>0$, it will be leftward. After the sensor recognizes the field signal and transmits the signal, the main control board will adjust the speed of the motor to drive the body to track. 


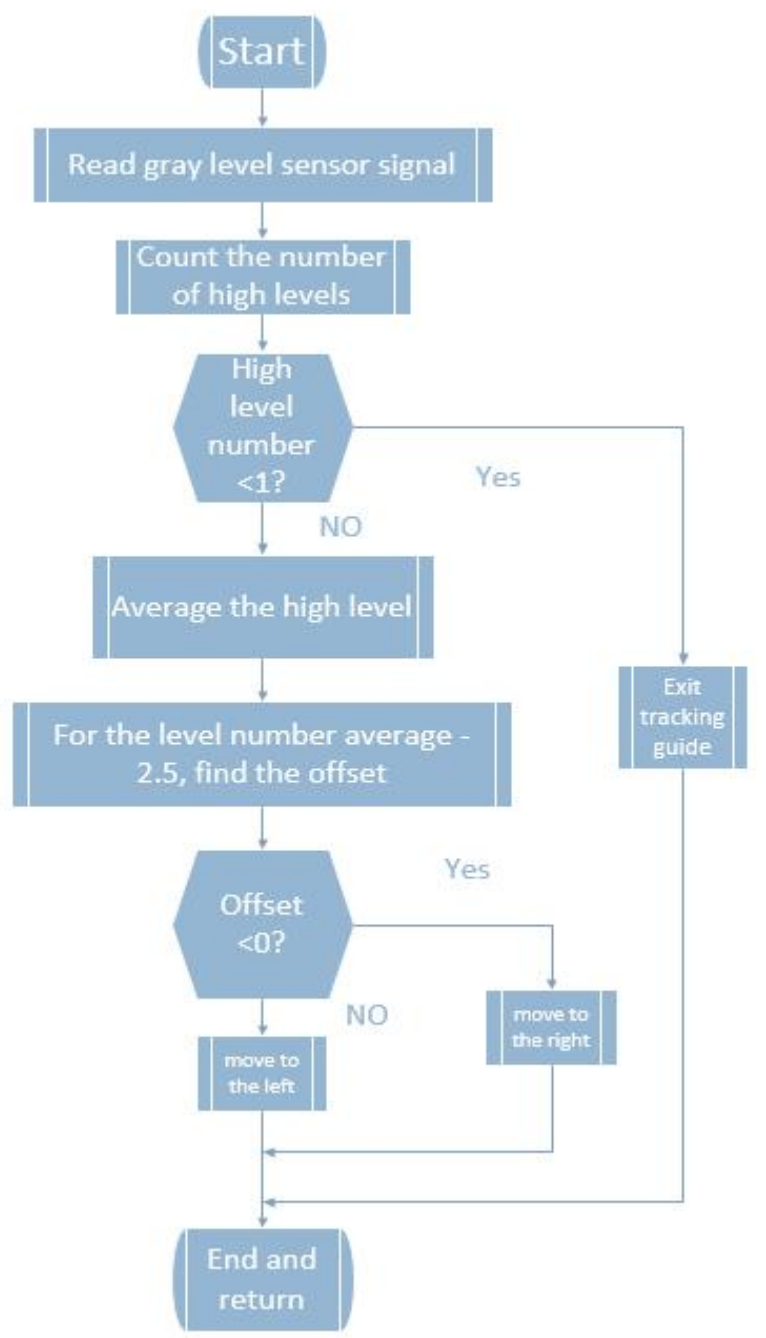

Fig10.Flow chart for tracking

Considering the versatility of the actual situation and the ease of writing of the algorithm, the robot uses four gray sensors as the sensing and detection system of the tracking module. The high level -2.5 in the above figure is also based on the number of the four gray sensors. . The advantage is that it can ensure the consistency of the detection signal and the actual body position, and it is also convenient for the detection signal and control. 


\subsection{Grab module}

This module is mainly composed of a mechanical arm and a mechanical claw. The mechanical arm is mainly used to expand the grasping range and the flexibility of the activity. The mechanical claw is mainly used to grasp the object. The specific structure of the mechanical claw can be based on the grasping target The external shape and size of the object depend on the specific grasping action and path planning, which requires a corresponding algorithm.

\subsection{Power module}

The current power supply method of the handling robot is mainly battery-powered. Considering that the handling robot often carries heavy objects and the travel distance is too long, it needs regular charging. At the same time, the battery is required to be charged quickly, saving charging time and improving work efficiency. Therefore, there are the following basic requirements for the selection of batteries for handling robots [13].

(1) Reliable and durable, able to realize uninterrupted handling robot, continuous work throughout the day, effectively improve the working efficiency of handling robot;

(2) The charge-discharge ratio is large, which can realize rapid charging with a large current of 1-4 times;

(3) Long working life;

(4) Small size and light weight, effectively reduce the weight of the vehicle, improve the load capacity of the handling robot, and increase the loading space;

At present, the batteries that are mainly used in handling robots are mainly nickelcadmium batteries, nickel-metal hydride batteries, lithium batteries, and lead-acid batteries. Lead-acid batteries are inexpensive and easy to manufacture, but due to their high weight and large size, they are not suitable for handling robots. The nickelcadmium battery is quick and practical to charge, but it will have a serious "memory effect" after being charged many times. It is not suitable for practical use. The improvement of the nickel-cadmium battery of the nickel-hydrogen battery is not obvious. The "memory effect" is not obvious, the life is long, but the high temperature resistance If the battery life is poor, the life will be reduced quickly, and the internal resistance of the battery will increase. Overcharging has a great impact on battery life and has certain risks. The lithium battery is small in size, high in energy density, convenient and fast to charge, and has a long life. Therefore, this article chose to use the lithium battery as the power supply for the handling robot. 


\section{Comprehensive structure display}

\subsection{Mechanical structure}

The whole car body is assembled by simple sheet metal parts made of aluminum alloy, which has the advantages of simple structure, easy implementation and low cost. As shown in Figure 11, it is a three-dimensional display of the overall structure.

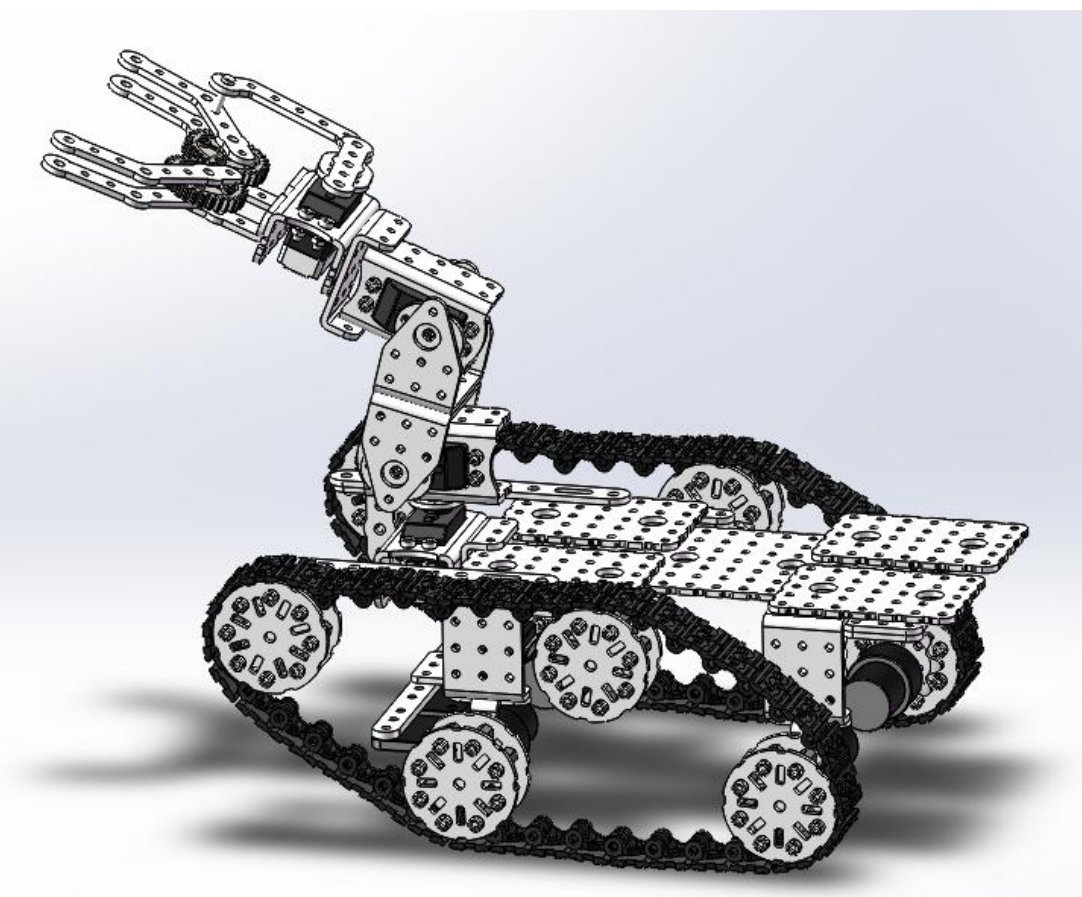

Fig11.Comprehensive 1 mechanical structure

\subsection{System software structure}

Figure 12 shows the flow diagram of the entire intelligent robot's active program. After receiving the task, the robot enters the program initialization, and then traces to the target location according to the trajectory line, grabs the object through the mechanical arm claw, and puts it into the cargo box, which can carry several objects at the same time, improving the efficiency of carrying. Then turn around and perform the tracking function again, move to the unloading location, and continue to unload the objects from the cargo box to the designated task location. 


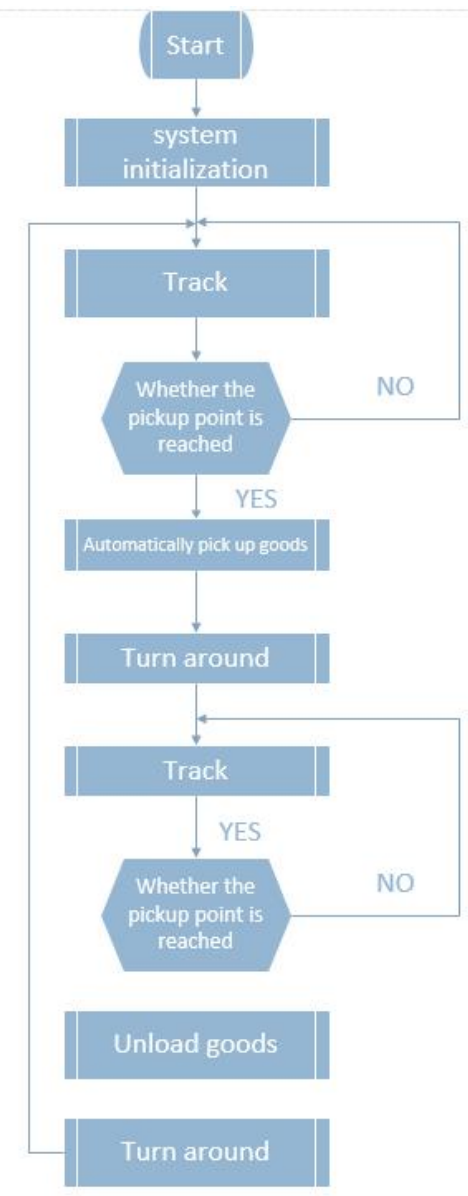

Fig12.Main program diagram

\section{Conclusion}

Research and design and development of an intelligent automatic identification tracking handling robot, including mechanical parts and control systems. The truck can automatically grasp and transport multiple objects at one time through mechanical arm claws, cargo boxes, etc. It can identify automatic tracking through the tracking module, and realize autonomous transportation operations as a whole.

The robot can be expanded and improved, through wireless data transmission, it can be used in factories, warehouses and other occasions that require automatic access and automatic classification., especially in dangerous places that cannot be entered by humans, replacing existing labor for cargo handling and sorting, It can be further expanded for cleaning in the industrial field. The successful development and 
promotion of the miniature intelligent handling robot can greatly reduce the labor intensity of the workers, improve the operation efficiency, and reduce the labor cost, which will have important practical significance.

\section{Acknowledgements}

This work was supported by the grant of Hubei Provincial Department of Education (D20191105).

\section{References}

1. Guicheng Du, Jiandong Wu, Research on Global Robot Industry Status and Trends and Forecast of China's Robot Industry [J]. Dongfang Electric Review, 28(112), 4-10(2014).

2. Hangyu $\mathrm{Xu}$, Design and implementation of control system for warehouse handling robot [D], 2017.

3. Wang Li, Lu Puwei, Lin Hailiang, et al. Design and implementation of a warehouse logistics handling robot control system[J]. Packaging \& Food Machinery, 2019, 37(003):24-29,33.

4. Wenping Wang, Tianpei Li, Gensheng Feng, Design and implementation of an intelligent handling robot [J].Computer Measurement and Control, 019(002), 395-398(2011).

5. Yin Qin, Chao Li, Zhiyong Zhang, A multi-mobile handling robot system structure for engineering applications [J]. Journal of Central South University: Natural Science Edition, (S2), 21-27(2013).

6. Farshad A, Jose E, Benjamin B , et al. Mona: an Affordable Open-Source Mobile Robot for Education and Research[J]. Journal of Intelligent and Robotic Systems, 2018.

7. Xu J X, Guo Z Q, Lee T H . Design and Implementation of Integral Sliding-Mode Control on an Underactuated Two-Wheeled Mobile Robot [J]. IEEE Transactions on Industrial Electronics, 61(7):3671-3681(2014).

8. Xiansheng Qin, Xuefeng Zhang, Xiaoqun Tan, Review of research on mammal-like legged robots [J]. China Mechanical Engineering, 024(006):841-851(2013).

9. Vu Q H, Kim B S, Song J B. Autonomous stair climbing algorithm for a small fourtracked robot $[\mathrm{C}] / / 2008$ International Conference on Control, Automation and Systems. IEEE, 2008.

10. Pandey A . Path planning navigation of mobile robot with obstacles avoidance using fuzzy logic controller[C]// 2014 IEEE 8th International Conference on Intelligent Systems and Control (ISCO). IEEE, 2015.

11. Petrus Gijsbertus Maria Centen, Friedrich Heizmann, Steffen Lehr. Image sensor and method for reading out pixels of the image sensor [J]. Nature, 384(6608):458-460.2(2012).

12. Han J. From PID to Active Disturbance Rejection Control [J]. IEEE Transactions on Industrial Electronics, 56(3):900-906(2009).

13. Hua Fan, Qiuxia Chen, Lihui Ni. Design of Lithium Battery BMS Management System for Mobile Robots[J]. Single-chip Microcomputer and Embedded System Application, 2019, 019(002):73-76. 